Nutr. Dieta 1966;8:I-IV

\title{
Contents, Vol. 7, 1966
}

\section{Nutritio et Dieta}

Europäische Zeitschrift für Ernährung und Diätetik

European Journal of Nutrition and Dietetics

Journal européen de nutrition et de diététique

\section{Editores}

G. Ågren, Uppsala

R. Ammon, Homburg/Saar

E. J. Bigwood, Bruxelles

R. Boller, Wien

B. Borgström, Lund

E. Le Breton, Paris

M. Coppo, Modena

H. D. Cremer, Giessen Ch. Darnaud, Toulouse M. Demole, Geneve M. J. L. Dols, Wassenaar N. Eeg-Larsen, Oslo

F. Fidanza, Perugia

H. Glatzel, Dortmund

W. Gloor-Meyer, Zurich

J. Gontzea, Bucarest

H. Gounelle, Paris J. J. Groen, Jerusalem

W. Halden, Graz

C. Den Hartog, Den Haag

E. HOFF-JøRGENSEN,

København B. Isaksson, Göteborg R. Jacquot, Paris H. Kraut, Dortmund J. Kühnau, Hamburg J.

Lederer, Louvain

F. Mancini, Roma

G. A. Martini, Marburg/Lahn

A. P. Meiklejohn, Edinburgh

0 . Mellander, Göteborg

H. G. Mogena, Madrid H. Mohler, Zurich A. Mosse, Paris R. Nicolaysen, Oslo G. Péquignot, Paris

F. A. Pezold, Berlin

P. Roine, Helsinki

H. Sarles, Marseille

T. F. S. M. Van Schaik, Den Haag

G. S. Schettler, Berlin

H. M. Sinclair, Oxford

J. C. Somogyi, Zurich

H. J. Thomasson, Vlaardingen 
F. J. Traissac, Bordeaux

L. Travia, Roma

G. P. Vecchi, Modena

G. Verdonk, Gand

F. Verzár, Basel

R. Wenger, Wien J. F. De Wijn, Zeist J. Yudkin, London

Redactores

E. Azérad, Paris

J. Trémolières, Paris

H. Kapp, Basel

K. A. J. Wretlind,

Stockholm N.Zöllner, München

1966

Vol. 8

BASEL (Schweiz)

S. KARGER

NEW YORK

INDEX

Bengtsson, G.: vide Jaarma, M.

Bigwood, E. J.: Senescence and Nutrition. An Important Sociological and Economic

Problem of Actuality to Nutritionists. - Alter und Ernährung. Ein wichtíges sozíologisches und wírtschaftliches, fur den Ernährungsforscher aktuelles Problem. - Senescence et nutrition. Un important problème économique et social, d'actualité pour le nutritionniste 226

Blazek, Z. and Dvorsky, A.: The Effect of $\alpha$-Tocopherol Acetate on Plasma Esterified Cholesterol in Patients with Chronic Liver Disease. - Eínfluß des a-Tokopherolazetats auf Plasmacholesterolester bei Kranken mit chronischen Leberstörungen. - L'action de Гacétate d' $\alpha$-tocophérol sur la fraction esterifiée du cholesterol plasmatique chez les malades souffrant d'affections hépatiques chroniques 100

Blumberger, W. und Glatzel, H.: Beiträge zur Physiologie der Gewürze und Würzstoffe. VII. Untersuchungen über den Mechanismus der Wirkung auf die Speicheldrüsen. The Physiological Effects of Spices and Condiments. VII. Investigations into the Me chanism of Action on Salivary Gland Function. - Contribution à la physiologie des condiments et des épices. VII. Recherches sur le mécanisme des effets sur les glandes salivaires $\quad 145$

Bramsel, H.: vide Wirths, W.

Brigant, L. et Lowy, R.: Effets des regimes gras au beurre et au saindoux sur la compo sition tissulaire du rat. Action du cholesterol et de la déficience en vitamines. - Wirkung fettreicher Díäten bei Verwendung von Butter oder Schweineschmalz auf die Gewebszusammensetzung der Ratte. Wirkung des Cholesterins und des Vítamínmangels. - The Effect of a Fat Regime of Butter and Lard on Tissue Composition in the Rat. The Action of Cholesterol and Vitamin Deficiency 31

Canzler, H. und Glatzel, H.: Untersuchungen über die Verdaulichkeit tierischer und pflanzlicher Proteinträger ohne und mit Fettzusatz. I. Der Ablauf der Magenentleerung. 
- Investigation into the Digestibility of Protein from Animal and Vegetable Sources with and without Additional Fat. I. The Course of Gastric Emptying. - Recherches sur la digestibilité des aliments protéiques d'origine animale ou végétale avec ou sans addition de graisses. I. Le déroulement de Гévacuation gastrique 49

Canzler, H. und Glatzel, H.: Untersuchungen über die Verdaulichkeit tierischer und pflanzlicher Proteinträger ohne und mit Fettzusatz. II. Die Passage durch Dunn- und Dickdarm. - Investigation into the Digestibility of Protein from Animal and Vegetable Sources with and without Additional Fat. II. The Passage through Small and Large In testine. - Etude sur la digestibilité des protéines d'origine animale et végétale en presence ou en absence de graisses. II. Le passage dans l'intestin grêle et le gros intestin .... 88 Clement, J. et Lemonnier, D.: Composition et structure des triglycérides de reserve du rat blanc soumis à des regimes à base de beurre. - Zusammensetzung und Struktur der Reserveglyzeride der weíßen Ratte unter Kostformen mit verschiedenem Gehalt an Butter. Composition and Structure of Stored Triglycérides in White Rats Subjected to Diets Based on Butter 22

Cremer, H.D.: vide Menden, E.

Dvorsky, A.: vide Blazek, Z.

Elwood, P. C: Utilization of Food Iron - An Epidemiologist's View. - Ausnutzung des Nahrungseisens in der Sícht des Epidemílologen. - Utilisation du fer alimentaire du point de vue de Гépidémiologiste 210

Fidanza, F.: Dietary Fat, Obesity and Coronary Heart Disease. - Nahrungsfett, Fettsucht und koronare Herzkrankheit. - Relations entre les lipides du regime, Fobésité et les atteintes des artères coronaires 200

Glatzel, H.: vide Canzler, H.

Griffaton, G.: vide Trémolières, J.

HÅkansson, I.; Holm, I. and Wretlind, A.: Studies of Complete Intravenous Alimen tation in Dogs. - Untersuchungen über ausschließlích intravenöse Ermährung beí Hunden.

- Etudes sur une alimentation intra-veineuse totale chez le chien 1

Hallberg, D.; Schuberth, 0. and Wretlind, A.: Experimental and Clinical Studies with Fat Emulsion for Intravenous Nutrition. - Experimentelle und klinische Unter suchungen mit Fettemulsionen für intravenöse Ernährung. - Etudes expérimentales et cliniques avec des emulsions graisseuses pour alimentation intraveineuse

Henricson, B.: vide Jaarma, M.

Holm, I.: vide HÅkansson, I.

Jaarma, Maire and Bengtsson, Gösta: On the Wholeseomeness of y-Irradiated Potatoes.

II. Feeding Experiments with Pigs. - Über die Zuträglíchkeit von $\gamma$-bestrahlten Kartoffeln.

II. Fütterungsversuche mit Schweinen. - Sur l'intégritê de pommes de terre irradiées aux rayons $\gamma$. II. Experiences de nutrition sur des pores 109

Jaarma, M.; Henricson, B. and Bengtsson, G.: On the Wholesomeness of y-Irradiated Potatoes. III. Feeding Experiments with Rats. - Über die Zuträglíchkeit $\gamma$-bestrahlter Kartoffeln. III. Fütterungsversuche mit Ratten. - Integrité des pommes de terre irradiées aux rayons $\gamma$. III. Experiences d'alimentation sur le rat 296 Jekat, F.: vide Kraut, $\mathrm{H}$.

Keller, W.: vide Wirths, W.

Kraut, H.; Jekat, F. und Pabst, W.: Ausnutzungsgrad und biologischer Wert des Pro teins der einzelligen Grünalge Scenedesmus obliquus, ermittelt im Ratten-Bilanz- 
Versuch. - Utilization and Biological Value of the Protein in the Unicellular Green Alga Scenedesmus obliquus as Shown by Balance Studies in the Rat. - Coefficient d'utilisation et valeur biologique des protéines de l'algue unicellulaire Scenedesmus obliquus appréciées par la mesure de bilan chez les rats 130

Kraut, H.: vide Wirths, W.

Lemonnier, D.: vide Clement, J.

Lowy, R.: vide Brigant, L.

Lowy, R.: vide Trémolières, J.

Menden, E. and Cremer, H.D.: A Laboratory Method for the Evaluation of Changes in Protein Quality. - Fine Laboratoriumsmethode zur Beurteilung von Veränderungen der Proteín-Qualität. - Une méthode de laboratoire pour évaluer les changements de qualité des protéines 188

Munro, H.N.: Relationship between Body Protein Synthesis and Protein Intake. -

Körpereiweíß-Synthese und Eíweíßzufuhr. - Relation entre la synthèse des protéines

corporelles et l'apport protéíque du regime 179

Pabst, W.: vide Kraut, H.

Passmore, R.: Caloric Expenditure in Man. - Kaloríenverbrauch des Menschen. - Dépense calorique chez Thomme 161

Pekkarinen, Maija and Roine, P.: Changes of Food Composition in Order to Improve

Nutritional Status. - Anderung der Zusammensetzung von Nahrungsmitteln zur Verbesserung des Nährwertes. - Modifications dans la composition alimentaire pour Tamélioration des états nutritionnels 235

Roine, P.: vide Pekkarinen, Maija

Schuberth, 0.: vide Hallberg, D.

Trémolières, J.; Lowy, R. and Griffaton, G.: Enzymatic Pathways of Ethanol

Metabolism at Normal and Toxic Levels. - Enzimatísche Stoffwechselwege des Aethanolabbaus beí normalen und toxischen Dosen. - Voies enzymatiques du métabolisme de Гéthanol aux doses normales et toxiques $\quad 282$

Wirths, W. und Bramsel, H.: Mengen und Geldwert der Eiweiß-, Fett- und Kohlenhydratkalorien am Nahrungsverbrauch aus Erhebungen von Wirtschaftsrechnungen 1958. - Quantities and Cost of Protein, Fat and Carbohydrate Calories in Food Consump tion Based on Household Accounts in 1958. - Quantité et prix de revient des calories protéiques, lipidiques et glucidiques de Talimentation d'après les données des statistiques économiques de 195861

Wirths, W.; Keller, W. and Kraut, H.: Work and Food. - Arbeit und Nahrung. -

Travail et alimentation $\quad 168$

Wretlind, A.: vide Håkansson, I.

Wretlind, A.: vide Hallberg, D.

Recommendations on the Nutritive Value of a Normal Diet for Adult Patients in Swedish Hospitals (February 1965). - Empfehlungen fur den Nährwert einer Normalkost für erivachsene Patíenten in schwedischen Spítälern (Februar 1965). - Recommendations sur la valeur nutritive d'un regime normal pour des malades adultes dans des hôpitaux suédois (février 1965) 81

Alle Rechte, insbesondere das der Übersetzung in ftemde SpГachen, vorbehalten. Ohne ausdrüekliche Genehmigung des Verlage $\beta$ ist es auch nicht gestattet, diesen Band Oder Teile 
daraus auf photomechanischem Wege (Photokopie, Mikrokopie) zu vervielfältigen.

(C)

Copyright 1966 by S. Karger AG, Basel

Printed in Switzerland

Cliches: Aberegg-Steiner \& Cie., AG

Druck: Lüdin AG, Liestal 